
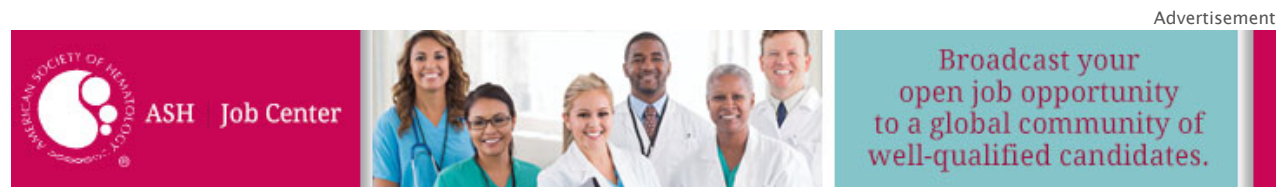

ASH Home Blood App My Folders Alerts RSS

Sign In

\title{
blood
}

Leading the way in experimental

and clinical research in hematology

search

Q

Advanced Search

Home | About Blood |

Authors

Submit to Blood

Subscriptions

Classifieds

Current Issue

First Edition

Collections

All Issues

Abstracts

Video Library

Home / November 15, 2013; Blood: 122 (21)

(1) No markup for post-processing

\section{Low-Dose Decitabine Vs Best Supportive Care In Older Patients With AML and Low Blast Counts: Results Of a Subgroup Analysis Of The Randomized Phase III Study 06011 Of The EORTC Leukemia Cooperative Group and German MDS Study Group}

Heiko Becker, MD¹, Stefan Suciu, PhD², Björn Rüter, MD*, ${ }^{1}$, Uwe Platzbecker, MD, PhD ${ }^{3}$, Aristoteles Giagounidis, MD*, 4 , Dominik Selleslag, MD*, ${ }^{2}$, Boris Labar, MD, PhD ${ }^{6}$, Ulrich Germing, MD*,7, Helmut R. Salih, MD*, ${ }^{8}$, Petra Muus, MD, PhD ${ }^{9}, K^{2}$ arl-Heinz Pflüger, MD*, ${ }^{2}$, Anne Hagemeijer, MD, PhD ${ }^{11}$, Hans-Eckart Schaefer, MD*, ${ }^{12}$, Frédéric Baron, MD, PhD ${ }^{13}$, Arnold Ganser, MD*, ${ }^{4}$, Carlo Aul, MD*15, Theo de Witte, MD*, ${ }^{16}$, Pierre W. Wijermans, $M^{17}$, and Michael Lübbert, MD $^{1}$

Author Affiliations

Article Figures \& Data

Info \& Metrics

E-Letters

PDF

\section{Abstract}

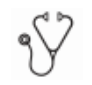

Introduction Decitabine has been approved for the treatment of myelodysplastic syndromes (MDS) in the United States and acute myeloid leukemia (AML) in older patients in Europe. The definitions of MDS and AML differ between the $F A B$ and WHO classification, mainly with regards to patients with 20 to $30 \%$ blasts in blood or bone marrow having MDS according to the $F A B$ classification (i.e. refractory anemia with excess blasts [RAEB] or RAEB in transformation), but AML according to the WHO. In the phase III trial 06011 , we compared low-dose decitabine with best supportive care (BSC) in patients $\geq 60$ years with MDS according to the FAB classification (Lübbert et al., J Clin Oncol. 2011;29:1987-96). Here, we examine trial 06011 for the efficacy and safety of decitabine in patients with AML according to WHO and low proliferation, i.e., blast counts of 20 to $30 \%$.

Patients and Methods Patients were randomly assigned to receive decitabine or BSC. Decitabine $15 \mathrm{mg} / \mathrm{m}^{2}$ was given intravenously over 4 hours every 8 hours for 3 consecutive days in 6 -week cycles, with a maximum of 8 cycles. Results were evaluated every 2 nd cycle. In case of complete remission (CR) at least 2 further courses were administered. Primary endpoint was overall survival (OS). Response rates (CR; PR, partial remission; HI, hematologic
November 15, 2013 Table of Contents

\section{$\leftarrow$ Previous}

Volume: 122

Issue: 21

Pages: 1452 - 1452

DOI: http://dx.doi.org/

\begin{tabular}{ll}
$\nabla$ Email & $\approx$ Save to My Folders \\
\hline C Correction Alert &
\end{tabular}

Article

Figures \& Data

Info \& Metrics

E-Letters 
improvement; PD, progressive disease), progression-free survival (PFS; time from random assignment to PD, relapse or death), AML-free survival (AMLFS; time from random assignment to $A M L$ according to $F A B$ [ $>30 \%$ bone marrow blasts] or death), and toxicity were secondary endpoints.

Results Applying the WHO criteria to the 233 patients enrolled onto the trial, 164 had MDS and 50 had AML with blast counts of 20 to $30 \%$. The remaining 19 patients were excluded from the present analyses. They comprised 14 patients with chronic myelomonocytic leukemia, 2 with $\mathrm{AML}$ and $\geq 40 \%$ blasts, and 3 with no blast counts available.

Among the AML patients, 27 were in the decitabine and 23 in the BSC arm. In both arms, the median age was 70 years. Of the patients in the decitabine arm, $59 \%$ received 3 or more treatment cycles. Response rates in the decitabine and the BSC arm were as follows: CR, $11 \%$ vs $0 \%$; PR, $11 \%$ vs $0 \%$; $\mathrm{HI}, 11 \%$ vs $0 \%$; and PD, $37 \%$ vs $74 \%$. Compared with the patients receiving BSC, those receiving decitabine had longer PFS ( $P=0.008$; Table 1). However, this did not translate into a significantly improved AMLFS or OS of the decitabine treated patients, although median OS was 9.8 months, compared to 5.9 months among patients receiving BSC only (Table 1). With regard to toxicity differences between the decitabine and BSC arms, grade 1-2 nausea was observed in $46 \%$ vs $14 \%$ and grade $3-4$ febrile neutropenia in $19 \%$ vs $0 \%$.

Among the MDS patients, those receiving decitabine $(n=78)$ had a longer PFS $(P=0.07)$ but similar AMLFS and OS compared to the patients receiving BSC only ( $n=86$; Table 1). The impact of decitabine on PFS, AMLFS and OS did not significantly differ between the AML and MDS patients (Table 1). Response rates among the MDS patients in the decitabine and BSC arms were as follows: $\mathrm{CR}, 14 \%$ vs $0 \%$; PR, $4 \%$ vs $0 \%$; HI, $18 \%$ vs $2 \%$; and PD, $23 \%$ vs $66 \%$.

Conclusions Our data point to the clinically relevant efficacy of decitabine given in the 3-day schedule among patients with AML and low blast counts, particularly by delaying progression or relapse. No impact of decitabine, compared to BSC or low-dose cytarabine, on OS in older patients with AML and 20 to $30 \%$ marrow blasts (median, 8.0 vs 6.1 months) has been previously also reported by Kantarjian et al. (J Clin Oncol. 2012;30:2670-7). In that study, decitabine was given with $20 \mathrm{mg} / \mathrm{m}^{2} /$ day on 5 days every 4 weeks; PFS was not presented. The prolonged PFS that we observe may be used for example as non-intensive bridge to allogeneic stem cell transplantation after reduced-toxicity conditioning. Due to the post-hoc nature of our analyses and the relatively small patient numbers, further studies appear warranted to fully establish the benefit of decitabine in AML patients with low blast counts.

Table 1

View inline | View popup
Related Articles

No related articles found.

\section{Articles by Suciu, S.}

Articles by Lübbert, M.

\section{Articles by Suciu, S.}

Articles by Lübbert, M.

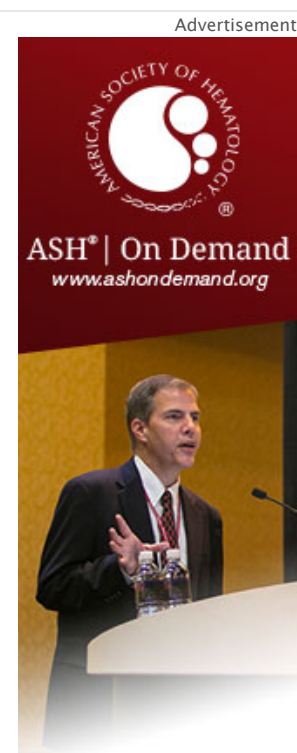

How will you treat your patients with the latest available agents? LEARN FROM THE EXPERTS

Disclosures: Rüter: Boehringer-Ingelheim: Employment. Platzbecker: Celgene: Honoraria, Research Funding; Novartis: Honoraria, Research Funding. Giagounidis: Celgene: Consultancy, Honoraria. Selleslag: Celgene: Consultancy; Novartis: Consultancy; Amgen: Consultancy. Baron: Genzyme: Honoraria.

- $\downarrow^{*}$ Asterisk with author names denotes non-ASH members.

- $\bigcup$ This icon denotes a clinically relevant abstract

(c) 2013 by The American Society of Hematology 


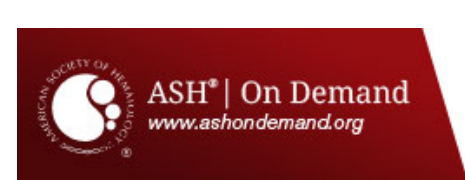

How will you treat your patients with the latest available agents? LEARN FROM THE EXPERTS.
Advertisement

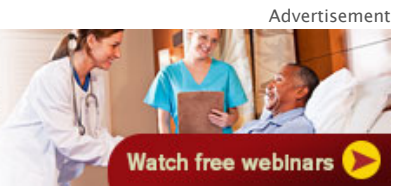

American Society of Hematology

2021 L Street NW, Suite 900, Washington, DC 20036

Phone 202-776-0544 | Fax 202-776-0545

Current Issue
First Edition
Topics
Collections
All Issues

All Issues

Abstracts
Leading the way in experimental and clinical research in hematology
Submit to Blood

Alerts

RSS

Blood App

Contact Us

Feedback
Information for:

Authors

Subscribers

Institutions/Librarians

Advertisers

ASH Privacy Policy

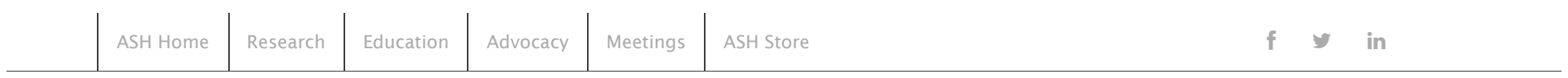

Copyright $\odot 2015$ by American Society of Hematology 